\title{
Adolescent girls' nutrition and prevention of anaemia: a school based multisectoral collaboration in Indonesia
}

\author{
Marion Roche and colleagues highlight lessons from a multisectoral project implementing \\ weekly iron supplementation for adolescent girls in West Java, Indonesia, which provides a scalable \\ model for reducing anaemia
}

I ron deficiency anaemia is estimated to be the single largest cause globally of morbidity and mortality in adolescent girls; it is expressed as disability adjusted life years. ${ }^{1}$ Disadvantages linked to iron deficiency anaemia include reduced academic potential; decreased wellbeing and productivity at home or in the community; and increased maternal and infant morbidity and mortality for adolescents who become pregnant. ${ }^{2}$ The World Health Organization recommends weekly iron folic acid supplementation (WIFAS) to reduce anaemia in adolescents aged 10-19 years and women of reproductive age, in regions where anaemia is a public health concern, affecting $>20 \%$ of females aged 15-49 years. ${ }^{2}$ In Indonesia, the national prevalence of

\section{KEY MESSAGES}

- A school-based iron supplementation project for adolescent girls in West Java, Indonesia provides a scalable model for anaemia reduction

- Existing platforms and policy frameworks for action help to catalyse multisectoral collaboration

- Political commitment from the highest policymaker of each sector or ministry is key, but local and institutional commitment, such as from each school principal, is also needed

- Capacity building is needed at all levels, but investments in strengthening individual and institutional relationships across sectors help to foster collaboration

- Data drive decisions and accountability, so harmonisation and collaboration on data collection, monitoring systems, and joint responsibility for, and ownership of, shared results, outcomes, and goals are key to engagement by all stakeholders anaemia among females aged 15-24 years is $18.4 \%{ }^{3}$; other surveys suggest a prevalence of $30 \%$ in adolescent girls. ${ }^{4}$ Screening data from junior high school students in West Java, the most populous province with 47 million inhabitants, ${ }^{5}$ indicated a prevalence of $>50 \%{ }^{6}$

Indonesia's Ministry of Health updated its national programme for anaemia prevention and control in adolescent girls and women of reproductive age (WIFAS policy) in 2016 to align more closely with WHO guidance (box 1). As part of the revision, it was suggested that an existing school health programme, Usaha Kesehatan Sekolah/Madrasah (UKS/M), should deliver the service ${ }^{7}$ (box 2). In Indonesia, adolescents rarely access preventive health services, but over $86 \%$ are enrolled in secondary school. Thus school based interventions are seen as ideal to reach adolescent girls, and are cost effective in other settings. ${ }^{9}$ Although the WIFAS policy had been revised, it had not been implemented, and practical guidance from the Ministry of Health was limited. To overcome this challenge, the ministry received technical and financial support from an international non-governmental organisation-Nutrition Internationalin 2015, to introduce a demonstration project for adolescent nutrition. This project worked through the school health programme to understand the roles, challenges, and opportunities inherent in this multisectoral approach, with the aim of designing a scalable programme.

This case study was developed in response to a global call for proposals by the Partnership for Maternal, Newborn, and Child Health. It aimed to identify factors for successful multisectoral collaboration for women's, children's, and adolescents' health. ${ }^{10}$ The methods used included document review and interviews with key informants to provide information for a working report, and a multistakeholder review of the findings of the working report (supplement 1).

Design of the demonstration project intervention The adolescent nutrition WIFAS demonstration project was conducted between 2015 and 2018. It aimed to show how the revised national WIFAS policy could be introduced through the UKS/M school health programme, supported by four ministries: Ministry of Health, Ministry of Education and Culture, Ministry of Religious Affairs, and Ministry of Home Affairs (box 3). The Ministry of Health selected the peri-urban and rural districts of Cimahi and Purwakarta, respectively, in West Java (fig 1), as implementation sites. The districts reported high rates of anaemia ( $>50 \%)$ and had also shown leadership in championing adolescent nutrition.

The demonstration project involved three key strategic components based on broader adolescent nutrition programme work by Nutrition International. This includes both WIFAS and nutrition education for adolescents ${ }^{11}$; see also, project timeline (supplement 2).

(1) Increasing awareness of, and securing government commitment to, the WIFAS project and adolescent health, in general. This to be achieved through implementation of policies, ensuring budget allocation for procurement and supply of IFA supplements, training, supportive supervision, and providing resources through joint advocacy meetings at national, provincial, and district levels.

(2) Improvement of the supply of commodities through skills building of Ministry of Health staff in forecasting, procuring, and supplying IFA for district health offices, and by strengthening supply chain management systems. Programme work also included provision of joint 
Box 1: Evolution of weekly iron folic acid supplementation (WIFAS) policies in Indonesia

- Since 1997, the government of Indonesia has had a mandate to introduce iron folic acid supplementation for adolescent girls, in order to reduce future maternal health complications. The original 1997 guideline recommended one supplement, once weekly for 16 weeks, and an additional supplement every day for 10 days of menstruation each month ${ }^{7}$

- This guidance was not aligned with the updated 2011 WHO guidelines for iron folic acid supplementation. ${ }^{2}$ These guidelines recommend one supplement weekly for 3 months and then 3 months without supplements ( 6 months total/year), or the option to implement the programme once weekly during the school semester, as aligned with the school calendar ${ }^{2}$

- In 2015, academics and nutritional experts, including Nutrition International, participated in technical consultations led by the Ministry of Health, to align national guidelines with theWHO recommendations. The revised Indonesian guideline recommends WIFAS of

- $60 \mathrm{mg}$ elemental iron $+400 \mu \mathrm{g}$ folic acid, once weekly for every week of the year. ${ }^{7}$ The formula recommended by WHO (60 mg elemental iron $+2800 \mu \mathrm{g}$ folic acid) was not affordable globally and on the job training for teachers and primary health facility (puskesmas) staff, and district officials from the health, education, and religious sectors. This aimed to improve knowledge of, and skills to prevent, anaemia, WIFAS consumption and counselling, and forecasting and reporting. Teachers distributed WIFAS to adolescent girls, supervised, and recorded consumption, and provided instructions on the prevention and management of side effects. Following best practice elsewhere, a fixed day approach was followed-for example, every Friday at assembly. ${ }^{12}$

(3) Increasing demand, and acceptability of the project to different stakeholders, through a behaviour change intervention strategy, including a branded campaign "Healthy, beautiful, and smart without anaemia" (fig 2). Campaign messages were based on formative research with adolescent girls, parents, health staff, teachers, and religious leaders. ${ }^{6}$
The behaviour change intervention strategy aimed to show that WIFAS and anaemia reduction would improve school performance and wellbeing of adolescent girls, rather than linking it to reproductive health (supplement 3).

Findings from the demonstration project could help to scale up the policy beyond these two districts. We estimate that the demonstration project might have contributed to preventing 4071 cases of anaemia, by reaching 52000 adolescent girls with the WIFAS scheme in the two districts. These figures are based on estimates from Nutrition International's Outcome Modelling for Nutrition Impact Tool $^{13}$; modelling based on the national prevalence of anaemia for women of reproductive age; and $27 \%$ cases of anaemia averted by WIFAS. ${ }^{14}$ Details of additional achievements provided by the endline evaluation measurements are shown in supplement 4. Below, we examine the different components of collaboration that contributed to this success.

\section{Box 2: Usaha Kesehatan Sekolah/Madrasah (UKS/M): national school health programme}

The UKS/M programme, established in 1976, aims to improve students' educational achievement by encouraging a healthy lifestyle and creating a healthy environment for students $^{8}$

The programme was updated in 1984, and endorsed by a joint regulation of four ministries: the Ministry of Education and Culture, Ministry of Health, Ministry of Religious Affairs, and Ministry of Home Affairs. Each sector has its own role and responsibilities for supporting UKS/M activities. These guidelines were updated in 2014. They provided a comprehensive list of activities for schools to adopt those most needed

The programme promotes intersectoral collaboration for school health among ministries with national, provincial, district, and subdistrict coordination teams. In schools, the head teacher and one or more teachers oversee UKS/M actions. Each school is expected to work with primary health facility (puskesmas) staff to carry out certain programme activities. In 2016 the national Ministry of Health requested that weekly iron folic acid supplementation should be one of the UKS/M activities
Enabling environment, political commitment, and local context

The multisectoral collaboration was driven by a change in policy. It was also enabled by an existing mandate for collaboration, political commitment at all levels, and by overall coordination across sectors by a non-governmental organisation (Nutrition International).

The Ministry of Health sent a letter to all heads of provincial and district health offices in Indonesia providing details of the WIFAS policy change. ${ }^{7}$ The updated anaemia prevention and control guideline suggested that IFA supplements should be delivered to schools through the existing UKS/M school health programme (box 2).

The letter clearly prioritised this initiative by provincial and district staff in the health sector. However, three other ministries (religious affairs, education and culture, and home affairs) needed to be involved when the Ministry of Health mandated delivery through the UKS/M programme. They had not yet received guidance from their own leadership (box 2).

Political commitment from these three sectors was generated through joint national, provincial, and district level meetings, facilitated by Nutrition International. The meetings aimed to raise awareness among all four sectors of the burden of anaemia for adolescent girls and the benefits of anaemia reduction. After these meetings, the district planning and development agency (Bappeda) agreed to contribute funds to train additional UKS/M teachers, beyond those covered by the Nutrition International project. In Indonesia, the importance of government endorsement of a project, including issuing circulars to national, provincial, and district/municipal areas, cannot be overestimated. In schools, better commitment from the staff to implement WIFAS activities was achieved when the head teacher issued an official letter.

"A key factor in the success of collaboration was the support from the head of the district and the legal standing of the project. Therefore, it can be merged into the district programme and consequently the budget will be secured." Key informant interview, district social welfare officer (May, 2018)

Although a programme and a written mandate for collaboration existed, assessment showed that the effectiveness of the UKS/M programme in schools was limited, with few activities taking place. 
Box 3: The roles of key partners and stakeholders in the weekly iron folic acid supplementation demonstration project

Ministry of Heath

- Develop programme planning and national coordination within the directorate general of public nutrition

- Facilitate coordination through UKS/M programme

- Ensure timely and adequate procurement of iron folic acid (IFA) supplements, in consultation with the district health offices, to ensure the availability of stocks at schools

- Support health staff and frontline health workers, through regular meetings and on the job training

- Strengthen monitoring and supervision of personnel; and review stocks and coverage of the IFA supplements

Ministry of Education and Culture and Ministry of Religious Affairs

- Support the programme by providing school and madrasah resources and infrastructure, including school staff and personnel

- Facilitate inclusion of health and nutrition education in the curriculum and activities for adolescents

- Include education sessions on a healthy school environment

- Encourage local government to improve the UKS/M infrastructure

Ministry of Home Affairs

- Facilitate implementation of the school health programme, including encouraging the district to develop local regulation for improving the UKS/M programme (including budget allocation)

- Support districts in establishing or strengthening the UKS/M secretariat

Nutrition International

- Technical support for procurement of IFA supplements and financial resources to fill any supply gaps

- Technical assistance through the provision of provincial and district coordinators

- Technical and financial support for advocacy meetings and training at provincial and district level; on the job training; development of training modules and behaviour change intervention materials; and evaluating the demonstration project with academic and research agency partners

\section{Adolescent girls}

- Participation in focus group discussions for the project's formative research

- Participation in the design, revision, and testing of behaviour change materials and nutrition education

- Peer leaders to support the focal point teachers

- Provide feedback on the programme during supportive supervision visits

This proved to be a key challenge with using $\mathrm{UKS} / \mathrm{M}$ to deliver WIFAS, and collaboration with schools needed to be strengthened. District coordinators, supported by Nutrition International, were crucial for revitalising the UKS/M programme and involving each sector, through extensive communication (box 3). Project stakeholders emphasised that this initial investment in the coordinators was essential for bringing together the relevant personnel across the multiple ministries.

Defined roles and responsibilities were described in the UKS/M. However, challenges emerged from the different structures in management, communication, and reporting between health and education sectors. This was due to different levels and timing of decentralisation across sectors, and to changes of structures in the education system during the project (supplement 5). The demonstration project worked with 244 schools that were accustomed to reporting to the provincial office. On the other hand, health services, including nutrition, were coordinated by, and reported to, their respective district/municipal health office. The project moved the expected lines of reporting, and the network of health facilities and district health offices now coordinated with schools. Secondary school management was moved to the provincial level in 2017 soon after the WIFAS project was implemented. This resulted in changes in leadership for the demonstration project in the education sector.

Although misalignment between the health and education system reporting lines was a challenge, decentralisation also had the benefit of fostering innovation by local champions. Under the decentralised system, primary and secondary schools have autonomy to prioritise which UKS/M activities to implement in their schools and school principals played a vital role in leadership of WIFAS activities. ${ }^{8}$ The district coordinators built relationships with school principals to create and maintain their interest in the project.

\section{Building capacity and relationships}

Joint capacity building succeeded in its original goals, but also had the unintended benefit of fostering relationships and communication. This proved invaluable for the collaboration. Project stakeholders across sectors agreed that the four day "training of trainers" course for district facilitators was the key entry point to collaboration.

The main lesson from workshops was that building relationships and trust among stakeholders was essential for improving collaboration. Such working relationships and communication across sectors were previously absent. Improving personal relationships was recognised by all as one of the main benefits of involvement in the project, in addition to improving knowledge of health and nutrition and identifying goals for the collaboration. Most informants used the term "silaturahmi" or "extending ties of friendships, fellowships, or fraternity" to describe their personal gain from the project (box 4). It was suggested that formal joint training and meetings should be followed up by informal networking, to improve collaboration and build trust across sectors. This was especially important at the district level, and revived the UKS/M programme in both districts, after a period of limited activities.

However, one challenge to sustaining relationships was to reduce the turnover of staff, especially in schools, as it was teachers who delivered the IFA tablets and 


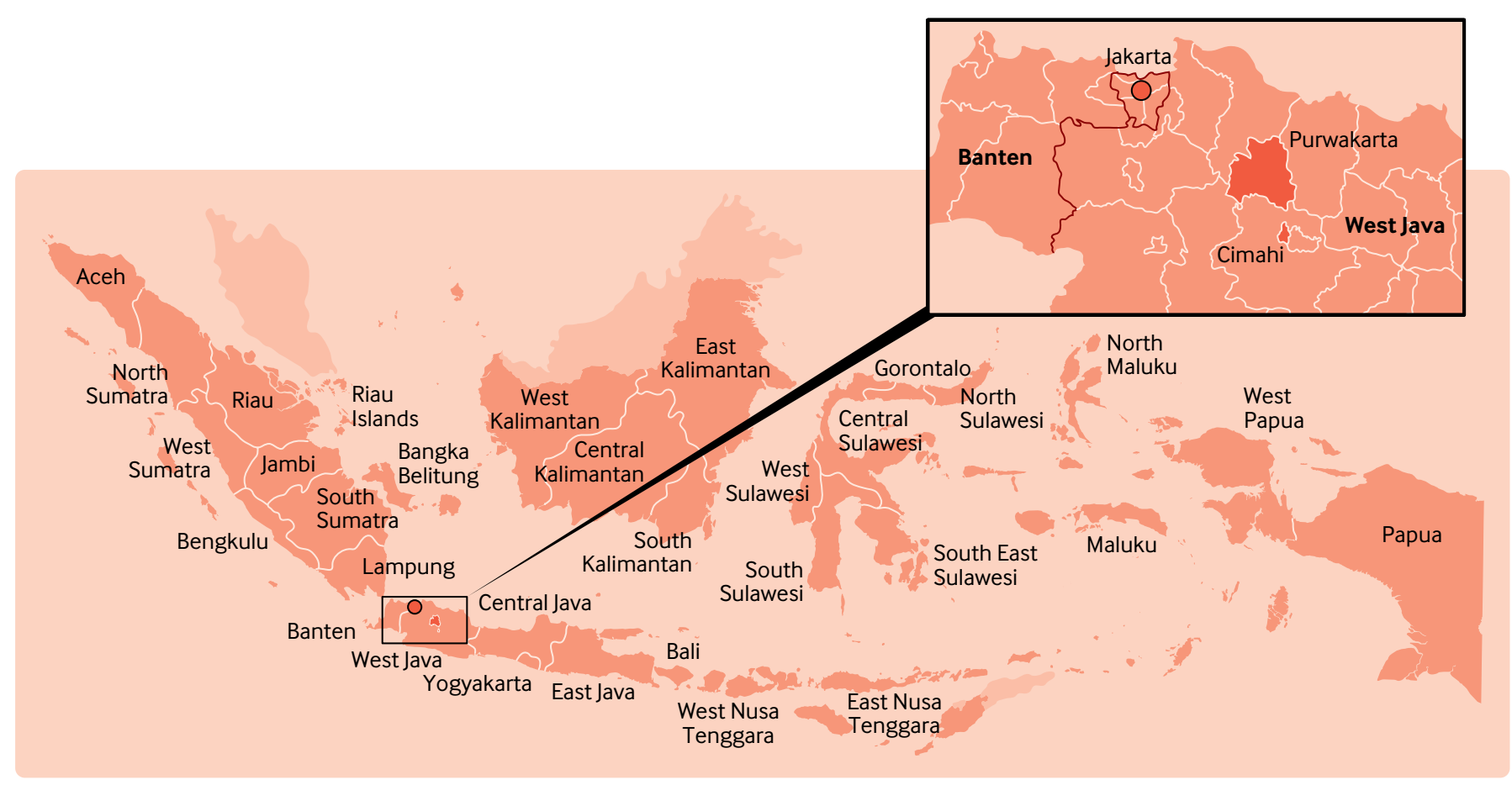

Fig 1 | Map of project demonstration sites

education to adolescent girls. Teachers could be easily transferred to other regions by the district or provincial education and religious affairs office. To reduce this problem the district health offices and Nutrition International conducted on the job training of teachers in schools, providing support and supervision. Some schools also used the WIFAS training manual to provide pretraining information for newly appointed teachers. Involving adolescent girls, such as those in junior Red Cross/Red Crescent groups, in distribution and reporting of WIFAS take up also helped the teachers.

Strengthening supply chain management and monitoring systems to mobilise resources Providing local data to show the need for, and potential of, the project was necessary for the engagement of participants. School enrolment data from the education office

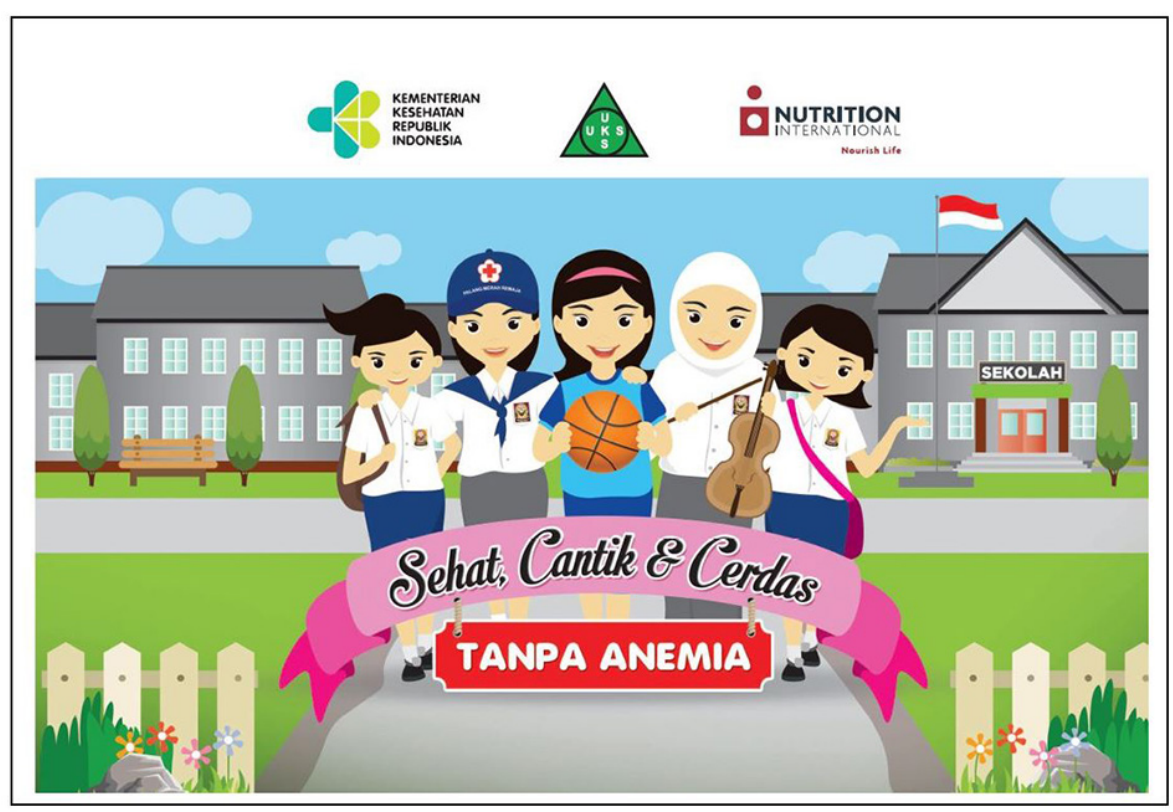

Fig 2 | Campaign image were essential for estimating commodity procurement by the district health offices. After initial resistance, data sharing became smoother when relationships were built:

"At the very beginning it was very difficult even to get a database of students to estimate the IFA stock for West Java... Now our head (provincial health office) is able to contact by phone her good friend at the provincial education office to get the data." Key informant interview, provincial health officer (May, 2018)

District budget constraints were also cited as a challenge to implementing the UKS/M programme. Little information about UKS/M financing at the various administrative levels is available; and without a monitoring and evaluation system, data are limited on the impact of the programme's activities. ${ }^{8}$ Participants outside the health sector considered that monitoring and evaluation should be carried out by the health sector. However, there was no mechanism for schools to share data about WIFAS activities before the demonstration project. The district coordinators helped to support harmonisation and reporting across systems and sectors and shared health data with all sectors involved. This helped to build interest in the progress being made, and accountability for implementation across sectors.

Use of evidence and local data involved participants and drove the decisions made. 
Box 4: The concept of silaturahmi and the importance of extending personal relations for collaboration

The Islamic-Indonesian term and concept of silaturahmi is very important in Indonesia for building personal relationships. It is based on the Islamic value of goodwill and fellowship, and the ability to extend personal ties of friendships to strengthen fraternity and mutual solidarity. To have a wide network of contacts is thought to provide many personal benefits. Thus, it is an important aspect of building relationships, contributing to successful collaboration across sectors for the WIFAS programme. This was expressed by one district stakeholder:

"Previously before the training of trainers we hardly knew each other, although we were all working under the district government. We very rarely had coordination meetings with other sectors, but after the training of trainers, we have been getting along very well ... it extends silaturahmi." Key informant interview, district religious affairs officer (May, 2018)

Budgeting for procurement of WIFAS commodities needed to align with district funding cycles. Thus support was needed for forecasting, with inputs required from both the health and education sectors. From 2015, the Ministry of Health planned to cover $20 \%$ of the cost of IFA tablets, increasing this to $30 \%$ by 2019 . Districts were expected to provide the rest. During the demonstration project Nutrition International covered the gap in supplements. Timely evidence based advocacy for WIFAS, use of resources, and a specific government budget will be required to sustain and scale up the WIFAS project.

\section{Engaging stakeholders at all levels}

The prevalence of anaemia was high in adolescent girls at the start of the project. Nevertheless, awareness of the risk and consequences of anaemia was low. This resulted in little demand for iron supplementation and little commitment among parents, teachers, and religious leaders. Iron supplements were generally perceived as being primarily for pregnant women. ${ }^{6}$

Evidence of the local burden of anaemia and the effectiveness of WIFAS, with appropriate description of the problem and benefits of WIFAS, was critical in securing support from all involved, including adolescents. The project highlighted the potential benefit of WIFAS and anaemia prevention for school performance:

"The district education office's mission is to educate children and young people... therefore we commit to working together and continue the [WIFAS] programme... if the children are not healthy, they will not be educated." Key informant interview, district education officer (June, 2018)

Adolescent girls have been underserved by the health system in Indonesia, so their cooperation is essential for the development of youth health services. ${ }^{15}$ Involving girls in developing the intervention was vital to the collaboration, following Nutrition
International's principle: "Nothing about her, without her." Nutrition education informed adolescent girls about their sexspecific nutritional needs, including iron, and the benefits of healthier diet and lifestyle choices. Teachers sought support from health staff to counsel girls who were rejecting supplements, but non-adherence was largely due to absence from school. Overcoming barriers to school attendance of girls, such as menstrual hygiene management facilities and early marriage, were challenges that remained. Furthermore, adolescent girls who did not attend school were beyond the reach of the UKS/M programme. Additional approaches to reaching vulnerable girls require further consideration.

Teachers were essential to success, as they distributed the supplements and counselled adolescent girls. Understanding anaemia and its effect on school performance and overall wellbeing, motivated teachers. However, with already high workloads, teachers needed to feel that the additional time required was worthwhile and recognised. Endorsement and acknowledgment by school principals and district health leadership was critical. This was achieved by sharing data showing progress in implementation in schools by assemblies and newsletters. This required project and district level results, compiled by the Ministry of Health, to be reported back to schools.

Parents may feel sceptical about interventions delivered outside the health system. They need to feel confident that products are safe and that the teachers delivering them are competent. For reassurance, parents were invited to meetings and sent letters stating the Ministry of Health endorsement of teachers, and details of the UKS/M programme. These were jointly produced by the Ministry of Health and the Ministry of Education and Culture. Schools that informed the parent school committee or met parents had better coverage and adherence to IFA consumption.

\section{Path to sustainability}

Although it is too early to confirm the sustainability of the project in the demonstration districts, some promising signs have been seen. These include establishment of the WIFAS project within UKS/M, revitalisation of the UKS/M, support from policymakers, and emergence of champions at every level. For example, project stakeholders at the district level have agreed to jointly pursue school operations grants and also funding from the universal health insurance programme. In Cimahi district, the collaboration has also expanded to include the district development planning agency (Bappeda), in support of implementation and to extend potential financial investments beyond the health sector.

Data from the project can stimulate interest, but it is still necessary to strengthen the internal monitoring system of the UKS/M programme, which currently depends on external support. Project participants were keen to improve coordination across sectors using mobile communication channels and to increase the quality of monitoring and evaluation. In Cimahi, the district health office and UKS/M are holding regular meetings on monitoring as a way to improve data quality and use. This is still in an early stage.

Importantly, scaling up of the WIFAS project is underway, based on the lessons learnt from the demonstration project. Expansion of external resources is taking place, with interest from both Canadian and Australian governments; while increasing financial commitments from district governments are being made. This case study shows that targeted investments in multisectoral collaboration will be critical for success.

The next steps should also focus on the $14 \%$ of adolescent girls who do not attend school in Indonesia, with gender inequalities, social norms, early marriage, and early childbearing making them all the more vulnerable to anaemia. Overcoming the limitations of this project to reach many of the vulnerable girls would be of interest to many district sectors. Given the many consequences of anaemia and gender inequality, concerted efforts for anaemia reduction are necessary to achieve the sustainable development goals.

\section{Conclusions}

This study of the WIFAS demonstration project in Indonesia highlights several key elements for successful collaboration: political commitment, enabling policy, shared goals, joint training and advocacy, 
building relationships and informal communication, and sharing data. The success in reaching adolescent girls in school and the estimated anaemia reduction, are greater than possible by any sector working alone. This article, describing the perspectives and experiences of multiple stakeholders, has illustrated challenges and opportunities that should inform scale up in Indonesia. It provides helpful insights for other countries aiming to reduce anaemia and improve nutrition for adolescent girls.

Multisectoral collaborations require resources and coordination. To further reach adolescents, it will be critical to build such collaborations that respond to the unique needs of countries.

The authors thank the many people who supported the preparation of this article, especially during the development of the case study working paper. At the national level, these include representatives from the Ministry of Health, Ministry of Education and Culture, Ministry of Religious Affairs, Ministry of Home Affairs, and the UKS/M secretariat, who we thank for their invaluable advice and direction for the WIFAS project and implementation, and support for developing the case study. We are also grateful to Elvina Karyadi in her former role as country director who worked with the Ministry of Health to establish and provide strategic leadership for the demonstration project. At the provincial level, we are grateful for the support of many people from the province health office, province education office, province religion office, province secretariat, and the UKS/M secretariat of West Java Province; and also the key staff from the district health office, district education office, district religion office, and district secretariat of Cimahi and Purwakarta districts for their valued cooperation during implementation of the demonstration project in those districts (2015-2018) and for providing information for the case study. For support during fieldwork the authors would like to thank staff from the Jatiluhur health facility (puskesmas) and staff and adolescent girls from Jatiluhur high school SMP1 in Purwakarta district, who willingly shared their experiences of the demonstration project. We also appreciate all the organisations that attended the case study multistakeholder dialogue workshop on 4 July 2018; and the research team led by Dr Rina Agustina, Dr Erfi Prafiantini, and Dr Budi Wiweko, from the Human Nutrition Research Cluster, Department of Nutrition, Faculty of Medicine University of Indonesia, for management of the baseline survey, formative research, and endline survey of the demonstration project.

Contributors and sources: MLR and LB conceived the outline of this paper; MLR drafted the first version, and revised it in consultation with LB. All authors reviewed and provided comments on the final draft. For the case study review supporting the findings of this article, INY wrote the draft working paper with inputs and final review from other authors; INY undertook key informant interviews; and LB drafted an initial summary of findings used for consultation with stakeholders. TSP and EKA provided technical supervision and support to the demonstration project, implementation and assessments, and the case study multistakeholder meeting. For the project and case study, SK provided overall strategic guidance and $A B$ technical guidance; DI provided advice and direction. All authors contributed intellectual content and approved the final version of this article for submission. MLR is the guarantor for this article.

Competing interests: We have read and understood BMJ policy on declaration of interests and declare the following interests: funding from the Partnership for Maternal, Newborn, and Child Health (PMNCH) secretariat (LB) and from Nutrition International (INY) for consultancy fees and related costs for undertaking the case study; employees of Nutrition International for implementing the project that is the focus of the case study (EKA, TSP, SK); and attendance at the forthcoming PMNCH partners' forum in December 2018 (MLR, LB, EKA, TSP, SK, DI, AB). The views expressed in this article are the authors and do not necessarily represent the views, decisions, or policies of the institutions with which the authors are affiliated.

Provenance and peer review: Commissioned; externally peer reviewed.

This article is part of a series proposed by the WHO Partnership for Maternal, Newborn and Child Health (WHO PMNCH) and commissioned by The BMJ, which peer reviewed, edited, and made the decision to publish the article. Open access fees for the series are funded by WHO PMNCH.

Marion Leslie Roche, senior technical adviser ${ }^{1}$

Louise Bury, independent consultant ${ }^{2}$

Isma Novitasari Yusadiredja, consultant ${ }^{3}$

Eriana Kartika Asri, programme officer ${ }^{4}$

Tutut Sri Purwanti, senior programme officer ${ }^{4}$

Sri Kusyuniati, country director ${ }^{4}$

Anjali Bhardwaj, regional manager ${ }^{1}$

Doddy Izwardy, director of public nutrition ${ }^{5}$

${ }^{1}$ Adolescents' and Women's Health and Nutrition,

Nutrition International, Ottawa, Canada

${ }^{2}$ Global Research Consultancy, Ipswich, UK

${ }^{3}$ Isma Novitasari Yusadiredjai, Bandung, Indonesia

${ }^{4}$ Nutrition International, Jakarta, Indonesia

${ }^{5}$ Directorate General of Public Health, Ministry of

Health, Jakarta, Indonesia

Correspondence to: M L Roche

mroche@nutritionintl.org

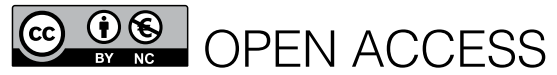

This is an Open Access article distributed under the terms of the Creative Commons Attribution IGO License (https://creativecommons.org/licenses/by$\mathrm{nc} / 3.0 /$ igo/), which permits use, distribution, and reproduction for non-commercial purposes in any medium, provided the original work is properly cited.

\section{Check for updates}

1 World Health Organization. Global accelerated action for the health of adolescents -AA-HA!): guidance to support country implementation. World Health Organization, 2017. http://www. who.int/maternal_child_adolescent/documents/ adolescents-health/en/

2 World Health Organization. Guideline: Intermittent iron and folic acid supplementation in menstruating women. World Health Organization, 2011. whalibdoc.who.int/ publications/2011/9789241502023_eng.pdf
3 National Institute of Health Research and Development. Ministry of Health, Republic of Indonesia. RISKESDAS. Ministry of Health, 2013.

4 World Health Organization. Prevention of iron deficiency anaemia in adolescents: role of weekly iron and folic acid supplementation. World Health Organization - Regional Office for South-East Asia, 2011. http://www.searo.who.int/entity/child adolescent/documents/sea_cah_2/en/

5 West Java Bureau of Statistics. West Java Province in figures, 2016. https://jabar.bps.go.id/publication/20 16/07/15/2297a52073f5f32eca26eb8a/provinsijawa-barat-dalam-angka-2016.html

6 Agustina R, Prafiantini E. Formative research for an improved iron folic acid supplementation program for school going adolescents in selected districts of West Java province in Indonesia. Department of Nutrition, Human Nutrition Research Cluster, Faculty of Medicine, Directorate of Research and Community Services, Universitas Indonesia \& Nutrition International, 2016

7 Ministry of Health. Anaemia prevention and control program in adolescent girls and women of reproductive age. Ministry of Health, Republic of Indonesia, 2016

8 Seameo Innotech. Analysis of the interface between Usaha Kesehatan Sekolah (UKS) programme and school based management (SBM). Bonn: Deutsche Gesellschaft für Internationale Zusammenarbeit (GIZ); 2016. http://www.fitforschool.international/wpcontent/ezdocs/SEAMEO_INNOTECH-GIZ_Analysis of UKS-SBM_Interface_in_Bandung 2017.pdf

9 Bundy D. School health and nutrition: policy and programs. Food Nutr Bull 2005;26(Suppl 2):S18692. 10.1177/15648265050262S209

10 Partnership for Maternal, Newborn \& Child Health. Success factors case studies from 12 countries announced. New Delhi, India, 2018. http://www.who. int/pmnch/media/news/2018/success-factors-casestudies/en/

11 Nutrition International. Women and girls' nutrition. https://www.nutritionintl.org/what-we-do/byprograms/women-girls-nutrition

12 World Health Organization. Weekly iron and folic acid supplementation programmes for women of reproductive age: an analysis of best programme practices. World Health Organization, 2011. http:// apps.who.int/iris/handle/10665/207489

13 Nutrition International. Outcome modelling for nutrition impact tool. https://www.nutritionintl.org/ knowledge-centre/omni

14 Fernández-Gaxiola AC, De-Regil LM. Intermittent iron supplementation for reducing anaemia and its associated impairments in menstruating women. Cochrane, 201110.1002/14651858. CD009218

15 Marcus R, Gupta-Archer N, Darcy M, Page E. Rigorous review. Girls' clubs, life skills programmes and girls' wellbeing outcomes. GAGE: 2017. https://www. gage.odi.org/sites/default/files/2017-10/GAGE\%20 Girls\%20Club\%20Report\%20FINAL.pdf

Supplement 1: Methods for the WIFAS project case study

Supplement 2: The project timeline Supplement 3: Behaviour change intervention strategy and campaign Supplement 4: Endline achievements Supplement 5: Decentralisation in the health and education sectors

See www.bmj.com/multisectoral-collaboration for other articles in the series.

Cite this as: $B M J$ 2018;363:k4541

http://dx.doi.org/10.1136/bmj.k4541 